\title{
ICP and Bullet Damage Analysis on Sheet-Metal and Wooden Boards
}

\author{
NATTAPONTANGTAWEE ${ }^{1,2}$, THEERAYUTMANEERUANGRIT ${ }^{1}$ and \\ WEERACHAIPHUTDHAWONG ${ }^{1 *}$
}

\author{
${ }^{1}$ Forensic Science Program, Department of Chemistry, Faculty of Liberal Arts and Science, \\ Kasetsart University, KamphaengSaen Campus, KamphaengSaen, NakhonPathom, 73140, \\ Thailand \\ ${ }^{2}$ The Department of Special Investigation (DSI), Ministry of Justice, 128, Laksi, Bangkok, \\ 10210, Thailand \\ faaswcp@ku.ac.th
}

Received 16 March 2015 / Accepted 18 April 2015

\begin{abstract}
The relationship between the distance of the gun and intended target using surface damage analysis have been studied. The sheet-metal and wooden boards were used as a target. The concentrations of $\mathrm{Pb}$ in Gunshot residues (GSR) were analyzed using Inductively coupled plasma (ICP). The analysis can be very useful in forensic science.
\end{abstract}

\section{Introduction}

During investigative proceedings of incidents involving the use of firearms, forensic expertise can provide key elements for the court to reach an opinion. In such context, particular attention is usually drawn to evaluative issues associated with firearms and related evidence such as gunshot residues (GSR) ${ }^{1,2}$. The analysis on the perforation of the bullet is also importance as at some crime scenes, bullets or their castings cannot be found with bullet damage and holes the only evidence available to determine firearm type and firing position. Upon arrival at the scene, it is the responsibility of the police to start the difficult job of determining the sequence of events of the case especially when shooting is involved ${ }^{3,4}$. When a gun is being fired, both burned and unburned powders from the propulsive charge as well as components from the primer, the bullet and the cartridge case form a cloud that may cause a roughly circular pattern around the bullet hole.

This work has attempted to study the $9 \mathrm{~mm}$ bullet damage on sheet-metal and wooden boards that presented the characterisation discharge distance and bullet type, such as standard or hollow-point. This allows the development of a database that may be used for general and forensic analysis of $9 \mathrm{~mm}$ bullet damage. 


\section{Experimental}

Samples were prepared in the following manner. Two type sheet-metal and wooden boards with $50 \times 50 \mathrm{~cm}$ dimension were chosen to make as shooting target (Figure 1). The semiautomatic Glock pistol, produced by GlockGes.m.b.H, located in Deutsch-Wagram, Austria, a Glock17 $9 \mathrm{~mm}$ gun with "Safe Action" was used for shooting test. Four bullets type were used to comparative study of damage wound. A Sony alpha APS-C Kit digital camera (made in Japan) was used to photographed the images.

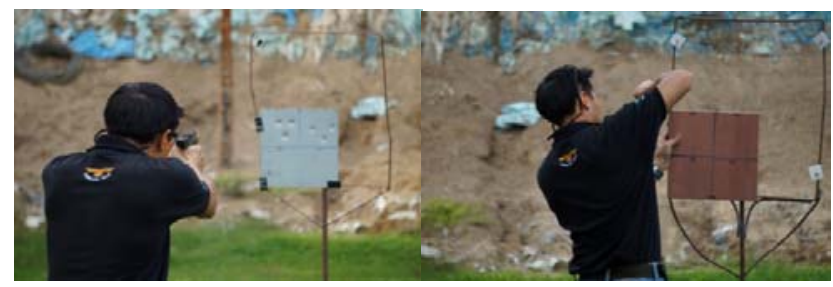

Figure 1. Sheet-metal and wooden boards with $50 \times 50 \mathrm{~cm}$ dimension

\section{Target material and firearm preparation}

Sheet-metal and wooden boards with $50 \times 50 \mathrm{~cm}$ were used for the shooting test. The stand bearing the target was then positioned at different distances from the shooter. The firearm, a $9 \mathrm{~mm}$ semi-automatic Glock pistol was prepared. Prior to firing on the target, at least three shots were fired to safe target area in order to ensure that the barrels will be coated with powder residues and to observe a damage on sheet-metal and wooden boards. The set-up for this experiment and all shooting were performed by specialized officers at the shooting gallery at Nakronpathom province.

\section{Shooting test}

The objective in this shooting test was to estimate the effective firing distance from the muzzle to the target. The distances were focused at ranges which was $50 \mathrm{~cm}$. The shots were tested at 90 degrees to the target. Each of the distance was repeated three.

\section{Inductively coupled plasma (ICP) check}

GSR samples were collected from the target. The shooting was conducted outdoors. GSR samples and calibration solutions were placed on Q- tip cotton swabs (a pair of swabs for each sample and standard), placed in $15 \mathrm{~mL}$ polypropylene screw-top tubes and dried overnight. ICP instrument (PerkinElmer) was used for analysis of lead in the solutions. Standard solution of $\mathrm{Pb}$, concentration of 10,20 and $40 \mathrm{mg} / \mathrm{L}$ were prepared before diluted to 1000,2000 and $4000 \mu \mathrm{g} / \mathrm{L}$ respectively. Sample preparation consisted of adding of $10 \%$ $(\mathrm{v} / \mathrm{v})$ nitric acid into $50 \mathrm{~mL}$ volumetric tube. The solutions were analyzed in unattended mode, employing the ASX- 500 (CETAC) autosampler and the Agilent ChemStation software feature allowing for sequential analysis of the samples.

\section{Results and Discussion}

The estimation of shooting distance was first made based on the direct and visual examination of the target. The result of four bullet type namely Lead head, Hole head, Couple 115 gain and Couple 124 gain of $9 \mathrm{~mm}$ bullets damage on sheet-metal and wooden boards that presented the characterisation discharge distance and bullet type, such as standard or hollow-point have been given in Figure 2 and 3. It is evident that bullet types were effected to damage holes. 


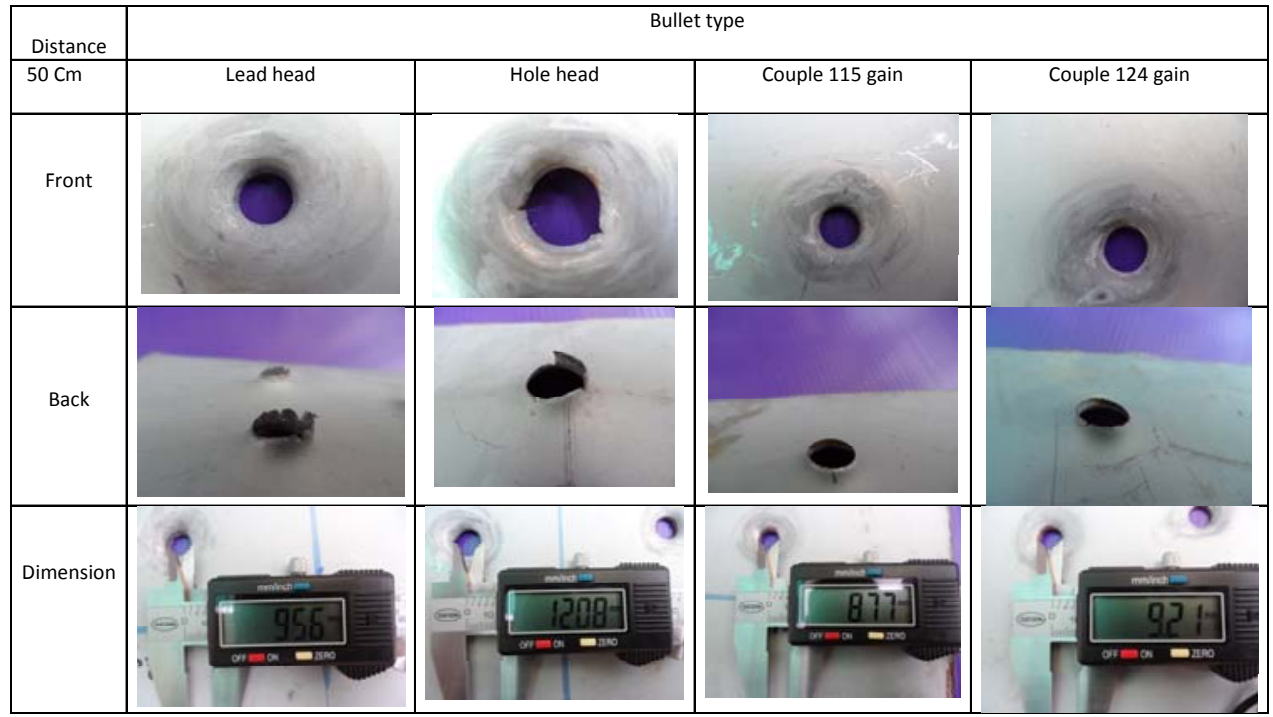

Figure $2.9 \mathrm{~mm}$ bullets damage on sheet-metal

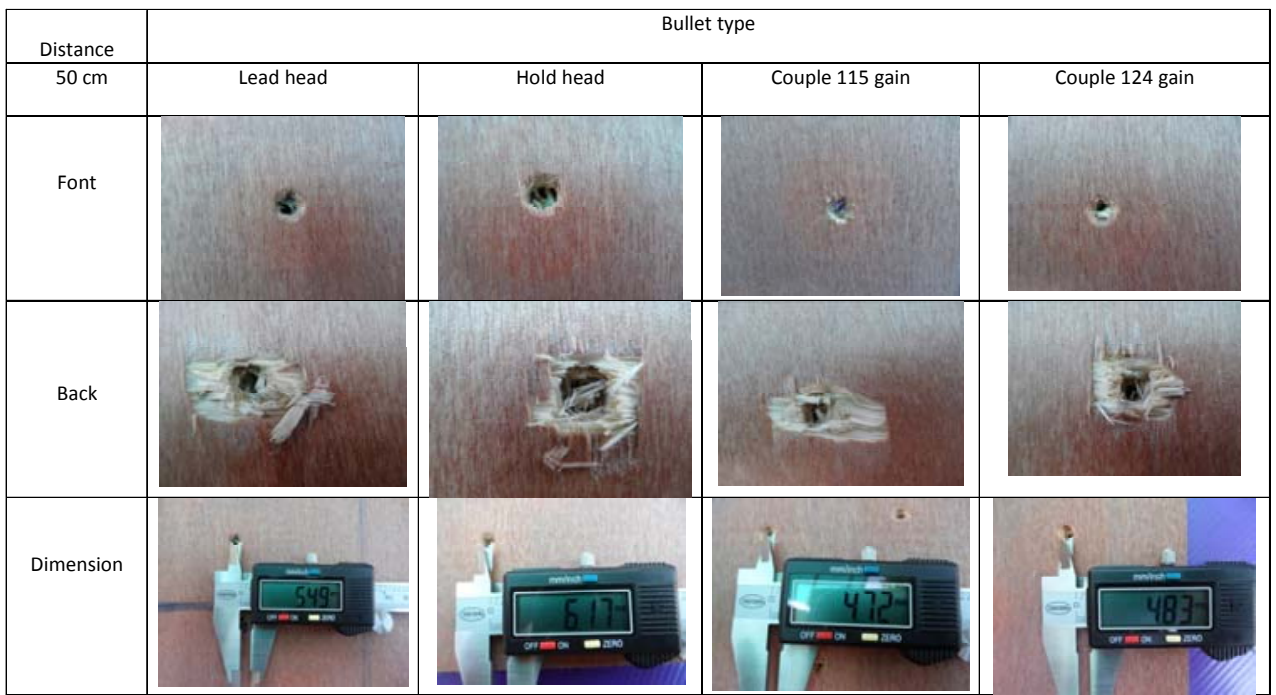

Figure 3. $9 \mathrm{~mm}$ bullets damage on wooden boards

A typical ICP analysis result of the chemical component of gunshot residues (GSR) at target were carried out. Samples were analyzed for the determination of only $\mathrm{Pb}$. The results of the quantitative analysis of those eight samples are presented as : Sheet-metal; Lead head, Hole head, Couple 115 gain and Couple 124 gain $/ 208 \mathrm{~Pb}=1.26,1.20,1.25,1.21 \mathrm{mg} / \mathrm{L}$ | Wooden boards; Lead head, Hole head, Couple 115 gain and Couple 124 gain $/ 208 \mathrm{~Pb}=$ 1.02, 1.20, 1.15, $1.17 \mathrm{mg} / \mathrm{L}$ respectively. The number of $\mathrm{Pb}$ in gunshot residues from each wound obtained from each shooting with different types of bullets shown increasing and depending on gain of bullet. Couple 124 gain bullet shown highest $\mathrm{Pb}$ value on both sheetmetal and wooden boards. The physical sheet-metal damage dimension holes from different types of bullet namely; Lead head, Hole head, Couple 115 gain and Couple 124 gain were 
9.56, 12.08, 8.77 and $9.21 \mathrm{~cm}$. The physical wooden boards damage dimension holes from different types of bullet, Lead head, Hole head, Couple 115 gain and Couple 124 gain were 5.49, 6.17, 4.72 and $4.83 \mathrm{~cm}$ respectively. It was found that both sheet-metal and wooden boards are shown significant different and can be used to identify type of bullet.

\section{Conclusion}

Information gained from this study can be used to estimate the muzzle-to-target distance for reconstruction of shooting test at $50 \mathrm{~cm}$. The result of four bullet type namely Lead head, Hole head, Couple 115 gain and Couple 124 gain of $9 \mathrm{~mm}$ bullets damage on sheet-metal and wooden boards that presented the characterisation discharge distance and bullet type, such as standard or hollow-point and Pd can be easily checked in this distance using ICP.

\section{Acknowledgements}

The authors are grateful to the Chemistry Department of Silpakorn University for ICP facilities.

\section{References}

1. Laurent M, Alain A, Cristina C and Eric B, J Forensic Legal Med., 2007, 14(3), 161164; DOI:10.1016/j.jcfm.2006.02.021

2. Druid H, Forensic Science International, 1997, 88(2), 147-162; DOI:10.1016/S03790738(97)00104-7

3. Katerina P, Francisco T, António J. M, Agostinho S and Teresa M, J Forensic Legal Med., 2009, 16(7), 407-410; DOI:10.1016/j.jflm.2009.04.003

4. David A L and Carin M V, J Forensic Legal Med., 2008, 15(5), 343-345;

DOI:10.1016/j.jflm.2007.10.009 